\title{
Resolutions for unit groups of orders
}

\author{
Sebastian Schönnenbeck ${ }^{1}$
}

Received: 11 November 2016 / Accepted: 19 December 2016 / Published online: 29 December 2016 (C) Tbilisi Centre for Mathematical Sciences 2016

\begin{abstract}
We present a general algorithm for constructing a free resolution for unit groups of orders in semisimple rational algebras. The approach is based on computing a contractible $G$-complex employing the theory of minimal classes of quadratic forms and Opgenorth's theory of dual cones. The information from the complex is then used together with Wall's perturbation lemma to obtain the resolution.
\end{abstract}

Keywords Homology of arithmetic groups - Maximal orders · Voronoi theory · Computational homological algebra

\section{Introduction}

Let $K$ be an imaginary quadratic number field with ring of integers $\mathcal{O}_{K}$. The integral homology of $\mathrm{GL}_{2}\left(\mathcal{O}_{K}\right)$ (or often rather the closely related group $\mathrm{PSL}_{2}\left(\mathcal{O}_{K}\right)$ known as a Bianchi group) is a widely studied concept in the literature (see for example $[4,9,20,22])$. In higher dimensions considerably less is known but there are still some results available (see $[10,11])$.

The group $\mathrm{GL}_{2}\left(\mathcal{O}_{K}\right)$ can be thought of as the unit group of the maximal $\mathcal{O}_{K}$-order End $_{\mathcal{O}_{K}}\left(\mathcal{O}_{K}^{2}\right)$ in the simple $\mathbb{Q}$-algebra $K^{2 \times 2}$. Thus questions about the homology of $\mathrm{GL}_{2}\left(\mathcal{O}_{K}\right)$ naturally generalize to questions about the homology of unit groups of general maximal orders in simple $\mathbb{Q}$-algebras. In this article we present an algorithm

Communicated by Graham Ellis.

Sebastian Schönnenbeck

sebastian.schoennenbeck@rwth-aachen.de

http://www.math.rwth-aachen.de/homes/Sebastian.Schoennenbeck

1 Lehrstuhl D für Mathematik, RWTH Aachen, Pontdriesch 14/16, 52062 Aachen, Germany 
to construct a free resolution in this very general setup. The approach generalizes the ideas used in [10] to compute the integral homology of $\mathrm{PSL}_{4}(\mathbb{Z})$ and [11] to compute the integral homology of $\mathrm{GL}_{3}$ over imaginary quadratic integers. Moreover the ideas presented here were recently used to compute maximal subgroups and presentations of these unit groups (see $[6,8]$ ). We start out by constructing a cell complex with suitable action in the Voronoi cone of positive definite forms (following [19] which itself is a generalization of [18]). The resulting cell complex does not directly yield a free resolution (seeing as there are usually nontrivial cell stabilizers), however, it can be used in a subsequent step together with Wall's perturbation lemma [24] to actually construct one.

The algorithm has been implemented (using a combination of $[5,13,15]$ ) in a few sample cases. In particular we were able to construct cell complexes for certain groups of type $\mathrm{SL}_{2}$ over imaginary quadratic integers having only finite cell stabilizers (where previously only complexes with infinite stabilizers were known) and we supplement some of the results of [11] by computing the torsion in the integral homology of $\mathrm{GL}_{3}\left(\mathcal{O}_{K}\right)$ for $K=\mathbb{Q}(\sqrt{-7})$ and $K=\mathbb{Q}(\sqrt{-1})$ in low dimensions.

The article is organized as follows. We start by reviewing some basic notions on maximal orders in simple $\mathbb{Q}$-algebras and the associated cone of positive definite forms. In Sect. 3 we then construct the cell complex of minimal classes and study its geometry. Section 4 is devoted to the construction of a free resolution starting from the non-free resolution we obtain from the cell complex and finally in Sect. 5 we present some examples of the results we were able to achieve using this method.

\section{Prerequisites}

In this section we want to review some facts on maximal orders in simple $\mathbb{Q}$-algebras and the cone of positive definite forms. A standard reference for the former is [21] and for the latter we refer to $[6,8]$.

\subsection{Maximal orders}

Let us first fix some notation. For the remainder of this article let $A$ denote a finitedimensional simple $\mathbb{Q}$-algebra. Then we have $A \cong D^{n \times n}$ for some finite-dimensional $\mathbb{Q}$-division algebra $D$ and $n \in \mathbb{N}$. Furthermore we will write $K=\mathrm{Z}(D)$ for the center of $K, \mathcal{O}_{K}:=\operatorname{Int}_{\mathbb{Z}}(K)$ for its ring of integers, and denote a maximal $\mathcal{O}_{K}$-order in D by $\mathcal{O}_{D}$.

Definition 1 An $\mathcal{O}_{D}$-lattice of rank $n$ is a finitely generated $\mathcal{O}_{D}$-submodule of the right $D$-module $V:=D^{n}$ containing a $D$-basis of $V$.

Remark 1 1. Let $L$ be some $\mathcal{O}_{D}$-lattice of rank $n$. Steinitz's theorem [21, Thm 4.13, Cor. 35.11] implies that there exist right $\mathcal{O}_{D}$-ideals $\mathfrak{c}_{1}, \ldots, \mathfrak{c}_{n}$ as well as a $D$-basis $e_{1}, \ldots, e_{n}$ of $V$ such that $L=e_{1} \mathfrak{c}_{1} \oplus \cdots \oplus e_{n} \mathfrak{c}_{n}$. The Steinitz invariant of $L$ is defined to be the class $\operatorname{St}(L):=\left[\mathfrak{c}_{1}\right]+\cdots+\left[c_{n}\right]$ in the group of stable isomorphism classes of right $\mathcal{O}_{D}$-ideals. 
2. If $n \geq 2$ two lattices $L_{1}, L_{2}$ of equal rank are isomorphic if and only if $\operatorname{St}\left(L_{1}\right)=$ $\operatorname{St}\left(L_{2}\right)$. In particular if $\operatorname{St}\left(L_{1}\right)=[\mathfrak{c}]$ we have $L_{1} \cong \mathcal{O}_{D}^{n-1} \oplus \mathfrak{c}$.

3. The endomorphism ring $\operatorname{End}_{\mathcal{O}_{D}}(L)=\{X \in A \mid X L=L\}$ is a maximal order in $\operatorname{End}_{D}(V) \cong A$ and any maximal order in $A$ is of this form [21, Cor. 27.6].

\subsection{The cone of positive definite forms}

Taking the scalar extension of our simple $\mathbb{Q}$-algebra $A$ with the reals yields a semisimple $\mathbb{R}$-algebra $A_{\mathbb{R}}:=A \otimes \mathbb{Q} \mathbb{R}$ which is therefore isomorphic to a direct sum of matrix rings over $\mathbb{R}, \mathbb{C}$ and $\mathbb{H}$ (the quaternions), respectively. We resort to the notation of [8] and set $d^{2}:=\operatorname{dim}_{K}(D)$. Moreover let $s$ be the number of real places of $K$ which ramify in $D, r$ the number of real places of $K$ which do not ramify in $D$, and $t$ the number of complex places of $K$. Having fixed this notation we know that

$$
D_{\mathbb{R}}:=D \otimes \mathbb{Q} \mathbb{R} \cong \bigoplus_{i=1}^{s} \mathbb{H}^{d / 2 \times d / 2} \oplus \bigoplus_{i=1}^{r} \mathbb{R}^{d \times d} \oplus \bigoplus_{i=1}^{t} \mathbb{C}^{d \times d}
$$

On this algebra we define the involution * (which is canonical up to the choice of the above isomorphism) componentwise to be transposition on the matrix rings over $\mathbb{R}$ and transposition and entrywise conjugation (complex or quaternionic, respectively) on the matrix rings over $\mathbb{C}$ or $\mathbb{H}$. This definition yields in the usual way a map

$$
\dagger: D_{\mathbb{R}}^{m \times n} \rightarrow D_{\mathbb{R}}^{n \times m}
$$

via transposition and applying * componentwise, in particular we get an involution on $A_{\mathbb{R}} \cong D_{\mathbb{R}}^{n \times n}$.

Definition 2 1. Let $\Sigma:=\left\{F \in A_{\mathbb{R}} \mid F^{\dagger}=F\right\} \subset A_{\mathbb{R}}$ denote the $\mathbb{R}$-subspace of $\dagger$-Hermitian elements of $A_{\mathbb{R}}$.

2. On $\Sigma$ we may define a positive definite bilinear form via

$$
\left\langle F_{1}, F_{2}\right\rangle:=\operatorname{Tr}\left(F_{1} F_{2}\right)
$$

where $\operatorname{Tr}$ indicates the reduced trace of the semisimple $\mathbb{R}$-algebra $A_{\mathbb{R}}$.

3. $\mathcal{P}$ will denote the cone of positive definite elements in $\Sigma$ :

$$
\mathcal{P}:=\left\{\left(q_{1}, \ldots, q_{s}, f_{1}, \ldots, f_{r}, h_{1}, \ldots, h_{t}\right) \in \Sigma \mid q_{i}, f_{j}, h_{k} \text { positive definite }\right\}
$$

The elements of $V_{\mathbb{R}}:=V \otimes_{\mathbb{Q}} \mathbb{R}$ correspond to elements of $\Sigma$ in the sense that for each $x \in V_{\mathbb{R}}:=V \otimes \mathbb{Q} \mathbb{R}$ we have $x x^{\dagger} \in \Sigma$.

Lemma 1 [8, Lemma 3.2] Let $F \in \Sigma$, then $F$ defines a quadratic form on $V_{\mathbb{R}}$ via

$$
F[x]:=\left\langle F, x x^{\dagger}\right\rangle, x \in V_{\mathbb{R}} .
$$

This form is positive definite if and only if $F \in \mathcal{P}$. 
With this lemma in mind we shall also refer to the elements of $\Sigma$ as 'forms'.

Lemma 2 1. $\mathcal{P}$ is an open subset of $\Sigma$.

2. Let $F_{1}, F_{2} \in \mathcal{P}$ then $\left\langle F_{1}, F_{2}\right\rangle>0$.

3. For all $F_{1} \in \Sigma-\mathcal{P}$ there exists $0 \neq F_{2} \in \overline{\mathcal{P}}$ with $\left\langle F_{1}, F_{2}\right\rangle \leq 0$, where $\overline{\mathcal{P}}$ denotes the topological closure of $\mathcal{P}$.

Proof 1. This is well known.

2. We may prove this componentwise. But then the spectral theorem holds and we may assume $F_{1}$ to be a diagonal matrix with positive real entries. In this case the assertion is obvious.

3. Let $F \in \Sigma-\mathcal{P}$. In this case the form which $F$ defines on $V_{\mathbb{R}}$ is not positive and there exists some $0 \neq x \in V_{\mathbb{R}}$ with $\left\langle F, x x^{\dagger}\right\rangle \leq 0$. Now $x x^{\dagger}$ is positive semidefinite and therefore in the topological closure of $\mathcal{P}$.

The preceding lemma shows that $\mathcal{P} \subset \Sigma$ constitutes a self-dual cone in the sense of [19].

\section{The CW-complex of well-rounded forms}

We keep the notation from the previous section and are now prepared to construct (for a given unit group of a maximal order) a cell complex with a suitable action. The cell complex is constructed by decomposing the cone $\mathcal{P}$ into so called minimal classes which were first introduced in [1] which is also our primary source.

\subsection{Minimal classes}

We will first introduce the notion of minimal classes which will define the desired cell structure. Most of the paragraph will closely follow the ideas of [8]. For the remainder of this section let $L$ denote a fixed $\mathcal{O}_{D}$-lattice of $\operatorname{rank} n, \Lambda:=\operatorname{End}_{\mathcal{O}_{D}}(L)$ its ring of endomorphisms, and $\operatorname{GL}(L):=\Lambda^{*}$ the corresponding unit group.

Lemma 3 The set $M_{L}:=\left\{l l^{\dagger} \mid 0 \neq l \in L\right\}$ is discrete in $A_{\mathbb{R}}$ and admissible in the sense of [19], i.e. for each sequence $\left(F_{i}\right)_{i \geq 1} \subset \mathcal{P}$ converging to an element $F \in \overline{\mathcal{P}}-\mathcal{P}$ the sequence $\left(\min _{x \in M_{L}} F_{i}[x]\right)_{i \geq 1}$ converges to 0 .

Proof $L$ is discrete in $V_{\mathbb{R}}$, hence $M_{L}$ is discrete in $\Sigma$. Let now $F \in \overline{\mathcal{P}}-\mathcal{P}$ be a positive semidefinite form. It suffices to construct a sequence $\left(l_{i}\right)_{i} \subset L-\{0\}$ such that $\left(F\left[l_{i}\right]\right)_{i}$ converges to 0 . To do this we decompose $V_{\mathbb{R}}=\operatorname{rad}(F) \oplus U$ into the radical of the form defined by $F$ and an arbitrary complement $U$ (in particular $F_{\mid U}$ is positive definite). Now let $U_{\epsilon}$ be the open ball of radius $\epsilon$ around 0 in $U$ relative to the norm defined on $U$ by $F$. Then $\operatorname{rad}(F) \oplus U_{\epsilon}$ is convex, centrally symmetric around the origin and of infinite volume. Minkowski's lattice point theorem then implies that there is some $0 \neq l_{\epsilon} \in L \cap\left(\operatorname{rad}(F) \oplus U_{\epsilon}\right)$ and we have $F\left[l_{\epsilon}\right] \leq \epsilon$. We may therefore construct the desired sequence and the assertion follows. 
We will now give a short introduction to the notion of weights which was introduced in [1] and which allows us to construct several non-equivalent cell decompositions of the same space.

Definition 3 1. A weight $\varphi$ on $L$ is a $\operatorname{GL}(L)$-invariant map from the projective space $\mathbf{P}\left(D^{n}\right)$ to the positive reals with maximum 1 .

2. By $\varphi_{0}$ we will denote the trivial weight, i.e. $\varphi_{0}(x):=1$ for all $x$.

In what follows we will not strictly distinguish between a weight $\varphi: \mathbf{P}\left(D^{n}\right) \rightarrow \mathbb{R}_{>0}$ and the induced map $L-\{0\} \rightarrow \mathbb{R}_{>0}, l \mapsto \varphi(l D)$.

Definition 4 1. Let $L=e_{1} \mathfrak{c}_{1} \oplus \cdots \oplus e_{n} \mathfrak{c}_{n}$ be a lattice. To $0 \neq l=\sum_{i=1}^{n} e_{i} l_{i} \in L$ we associate the integral left $\mathcal{O}_{D}$-ideal $\mathfrak{a}_{l}:=\sum_{i=1}^{n} \mathfrak{c}_{i}^{-1} l_{i}$ and its integral norm $\mathrm{N}\left(\mathfrak{a}_{l}\right):=\left|\mathcal{O}_{D} / \mathfrak{a}_{l}\right|=\mathrm{N}_{K / \mathbb{Q}}\left(\mathrm{nr}\left(\mathfrak{a}_{l}\right)^{d}\right)$.

2. Let $x \in D^{n}$ and $0 \neq \lambda \in D$ arbitrary with $x \lambda \in L$. We define

$$
\mathrm{N}_{x}:=\mathrm{N}\left(\left[\operatorname{nr}\left(\mathfrak{a}_{x \lambda}\right)\right]\right)=\min _{I \triangleleft \mathcal{O}_{D},[\operatorname{nr}(I)]=\left[\operatorname{nr}\left(\mathfrak{a}_{x \lambda}\right)\right]} \mathrm{N}_{K / \mathbb{Q}}\left(\operatorname{nr}(I)^{d}\right) .
$$

3. For $x \in D^{n}$ we set $\varphi_{1}(x):=\mathrm{N}_{x}^{-2 /|K: \mathbb{Q}|}$.

Proposition 1 [8, Lemma 4.3] The function

$$
\varphi_{1}: \mathbf{P}\left(D^{n}\right) \rightarrow \mathbb{R}, \quad[x] \mapsto \varphi_{1}(x)
$$

is a well-defined weight.

We mention the strange looking weight $\varphi_{1}$ at this point as in some cases (e.g. when $D$ is an imaginary quadratic number field) it is in some sense more natural to work with $\varphi_{1}$ instead of $\varphi_{0}$ (see [7] for an exposition). For the remainder of this section we will now fix -in addition to the lattice $L$ - a weight $\varphi$.

Definition 5 1. Given $F \in \mathcal{P}$ we define the $L$-minimum of $F$ with respect to $\varphi$ to be

$$
\min _{L}(F):=\min _{0 \neq l \in L} \varphi(l) F[l] .
$$

2. The set of shortest vectors in $L$ with respect to $F$ and $\varphi$ is then denoted by

$$
\mathcal{S}_{L}(F):=\left\{l \in L \mid \varphi(l) F[l]=\min _{L}(F)\right\} .
$$

The following remark is essential for all computations with these definitions.

Remark 2 Let $F \in \mathcal{P}$. The set $\mathcal{S}_{L}(F)$ is finite, since

$$
\mathcal{S}_{L}(F) \subset\left\{0 \neq l \in L \mid F[l] \leq \frac{\min _{L}(F)}{\min _{0 \neq y \in L} \varphi(y)}\right\}
$$

which is a set of shortest vectors in a $\mathbb{Z}$-lattice and hence finite. The set is well-defined because $\varphi$ attains only finitely many values. 
We are now prepared to define the cell decomposition of $\mathcal{P}$ with respect to the lattice $L$ and the weight $\varphi$.

Definition 6 1. Let $F \in \mathcal{P} \cdot \mathrm{Cl}_{L}(F):=\left\{H \in \mathcal{P} \mid \mathcal{S}_{L}(H)=\mathcal{S}_{L}(F)\right\}$ is called the minimal class of $F$.

2. If $C=\mathrm{Cl}_{L}(F)$ is a minimal class we set $\mathcal{S}_{L}(C):=\mathcal{S}_{L}(F)$.

3. A minimal class $C$ is called well-rounded if $\mathcal{S}_{L}(C)$ contains a $D$-basis of $V$.

4. $F \in \mathcal{P}$ is called perfect if $\mathrm{Cl}_{L}(F)=\left\{a F \mid a \in \mathbb{R}_{>0}\right\}$.

Note that all these definitions depend on the choice of the lattice $L$ as well as the choice of the weight $\varphi$.

Remark $3 \mathrm{GL}(L)$ acts on $\mathcal{P}$ via $g F:=g F g^{\dagger}$ and this action respects the decomposition of $\mathcal{P}$ into the minimal classes. We therefore get a natural action of $\operatorname{GL}(L)$ on the set of minimal classes.

Lemma 4 [8, Lemma 5.3] Let $C \subset \mathcal{P}$ be a well-rounded minimal class and set $T_{C}:=$ $\sum_{x \in \mathcal{S}_{L}(C)} x x^{\dagger}$. Then $T_{C}$ is a positive definite form, $\operatorname{Stab}_{\mathrm{GL}(L)}(C)=\operatorname{Stab}_{\mathrm{GL}(L)}\left(T_{C}^{-1}\right)$, and the class $C^{\prime}$ is in the same $\mathrm{GL}(L)$-orbit as $C$ if and only if $T_{C}^{-1}$ and $T_{C^{\prime}}^{-1}$ lie in the same $\mathrm{GL}(L)$-orbit.

Thus it is computationally easy to construct stabilizers of minimal classes or check whether two minimal classes lie in the same GL $(L)$ orbit.

Remark 4 The above lemma directly implies that $\operatorname{Stab}_{\mathrm{GL}(L)}(C)$ is finite for any wellrounded class $C$.

\subsection{A closer look at the decomposition}

Let us now take a closer look at our decomposition and its geometry.

First of all note that $\mathcal{P}$ is a convex and thus contractible topological space. However we do not want to use $\mathcal{P}$ for our computations since we have already seen that we can only guarantee finiteness of cell stabilizers for well-rounded minimal classes. Let us introduce some additional notation.

Definition 7 1. We will use the notation $\Sigma^{\mathrm{wr}}:=\{F \in \mathcal{P} \mid F$ well-rounded $\}$ for the space of well-rounded forms.

2. $\Sigma_{=1}^{\mathrm{wr}}$ will denote the space of well-rounded forms whose $L$-minimum is 1 .

Since any form may be rescaled to have minimum 1 we will from now on think of well-rounded minimal classes as subsets of $\Sigma_{=1}^{\mathrm{wr}}$.

The following result shows us that $\Sigma_{=1}^{\mathrm{wr}}$ is still a suitable candidate to help us in constructing a free resolution.

Proposition 2 [23, Thm. 1] The space $\Sigma^{\mathrm{wr}}$ (and thus $\Sigma_{=1}^{\mathrm{wr}}$ ) is a (GL(L)-invariant) deformation retract of $\mathcal{P}$ and thus contractible.

As we already know about some finiteness results it is important to note that the action of $\operatorname{GL}(L)$ on the cells in $\Sigma_{=1}^{\mathrm{wr}}$ admits only finitely many orbits. To see this we need the following result of A. Ash. 
Theorem 1 [1, Thm.(ii)] The set $\Sigma_{=1}^{\mathrm{wr}} / \mathrm{GL}(L)$ is compact.

Corollary 1 Up to positive real homotheties and the action of $\operatorname{GL}(L)$ there are only finitely many perfect forms.

Proof Lemma 3 implies that [19, Lemma 1.6] holds. Hence the set of perfect forms in $\Sigma_{=1}^{\mathrm{wr}}$ is discrete. Together with Ash's aforementioned theorem this implies the assertion.

To show that this finiteness result also holds for the minimal classes of non-perfect forms we first need some further results.

Lemma 5 Let $C \subset \Sigma_{=1}^{\mathrm{wr}}$ be a minimal class. Then $C$ is convex.

Proof Let $F_{1}, F_{2} \in C$ and $\lambda \in[0,1]$. Then for all $l \in L$ we have

$$
\lambda \varphi(l) F_{1}[l]+(1-\lambda) \varphi(l) F_{2}[l] \geq \lambda \min _{L}\left(F_{1}\right)+(1-\lambda) \min _{L}\left(F_{2}\right)=1 .
$$

And equality holds exactly for $l \in \mathcal{S}_{L}\left(F_{1}\right)=\mathcal{S}_{L}\left(F_{2}\right)$. Hence $C$ is convex.

Definition 8 On the set of minimal classes we define the following partial ordering:

$$
C \preceq C^{\prime}: \Leftrightarrow \mathcal{S}_{L}(C) \subset \mathcal{S}_{L}\left(C^{\prime}\right)
$$

Obviously this partial ordering is compatible with the $\operatorname{GL}(L)$-action. In fact the following lemma shows that it coincides with the inclusion ordering arising from the cell decomposition.

Lemma 6 Let $C \subset \Sigma_{=1}^{\mathrm{wr}}$ be a minimal class and $\bar{C}$ its topological closure in $\Sigma_{=1}^{\mathrm{wr}}$. Then

$$
\bar{C}=\bigcup_{C^{\prime} \succeq C} C^{\prime} .
$$

Proof Let $\left(F_{i}\right)_{i} \subset C$ be a sequence converging to $F$. Since taking the $L$-minimum is continuous, $F$ will again have $L$-minimum 1 and attain this value on the elements of $\mathcal{S}_{L}(C)$. Hence by Lemma 3 we have $F \in \mathcal{P}$ and $\mathcal{S}_{L}(C) \subset \mathcal{S}_{L}(F)$ so $F$ lies in the above union. This shows that $\bigcup_{C^{\prime} \succeq C} C^{\prime}$ is in fact a closed set.

On the other hand let $F^{\prime}$ be in said union and $F \in C$. We set $F_{\lambda}:=(1-\lambda) F+\lambda F^{\prime}$ and see that $F_{\lambda} \in C$ for all $\lambda \in[0,1)$ since

$$
\varphi(l) F_{\lambda}[l]=\varphi(l)(1-\lambda) F[l]+\varphi(l) \lambda F^{\prime}[l] \geq(1-\lambda)+\lambda \varphi(l) F^{\prime}[l] \geq 1
$$

with equality if and only if $l \in \mathcal{S}_{L}(F)=\mathcal{S}_{L}(C)$. Hence there is a sequence of elements of $C$ converging to $F^{\prime}$ and the assertion holds.

Lemma 7 Let $C \subset \Sigma_{=1}^{\mathrm{wr}}$ be a minimal class. Then the topological closure of $C$ contains only finitely many perfect forms. 
Proof Since we already know that there are only finitely many perfect forms up to the action of $\operatorname{GL}(L)$ it suffices to show that for a given perfect form $F$ there are only finitely many $g \in \mathrm{GL}(L)$ with $g F g^{\dagger} \in \bar{C}$. Now the following holds

$$
g F g^{\dagger} \in \bar{C} \Leftrightarrow \mathcal{S}_{L}\left(g F g^{\dagger}\right) \supset \mathcal{S}_{L}(C) \Leftrightarrow g^{-\dagger} \mathcal{S}_{L}(F) \supset \mathcal{S}_{L}(C) \Leftrightarrow \mathcal{S}_{L}(F) \supset g^{\dagger} \mathcal{S}_{L}(C) .
$$

Now $\mathcal{S}_{L}(C)$ contains a basis for $V$ and thus $g^{\dagger}$ is uniquely determined by its values on $\mathcal{S}_{L}(C)$. Since $\mathcal{S}_{L}(C)$ and $\mathcal{S}_{L}(F)$ are finite there are only finitely many maps from $\mathcal{S}_{L}(C)$ to $\mathcal{S}_{L}(F)$ and therefore in particular only finitely many $g \in \mathrm{GL}(L)$ fulfilling the condition.

Note that [19, Prop. (1.6)] implies that there always is a perfect form in the closure of a minimal class.

Definition 9 Let $C \subset \Sigma_{=1}^{\mathrm{wr}}$ be a minimal class and $N:=\operatorname{dim}_{\mathbb{R}}(\Sigma)$.

1. The dimension $\operatorname{dim}_{\mathbb{R}}\left(\left\langle x x^{\dagger} \mid x \in \mathcal{S}_{L}(C)\right\rangle\right)$ is called the perfection rank of $C$, the codimension $N-\operatorname{dim}_{\mathbb{R}}\left(\left\langle x x^{\dagger} \mid x \in \mathcal{S}_{L}(C)\right\rangle\right)$ the perfection corank of $C$.

2. The affine space generated by $C$ will be denoted by $\operatorname{Aff}(C)$.

Lemma 8 Let $C \subset \Sigma_{=1}^{\mathrm{wr}}$ be a minimal class. Then $C$ is open in $\operatorname{Aff}(C)$.

Proof This a direct consequence of [19, Lemma (1.3)], which is applicable because of Lemma 3.

The following lemma shows that the perfection corank is actually a feasible way to determine the dimension of a minimal class.

Lemma 9 Let $C \subset \Sigma_{=1}^{\mathrm{wr}}$ be a minimal class. Then $\operatorname{Aff}(C)=\{F \in \Sigma \mid \varphi(l) F[l]=$ $\left.1 \forall l \in \mathcal{S}_{L}(C)\right\}$. Moreover the (affine) dimension of $\operatorname{Aff}(C)$ is precisely the perfection corank of $C$.

Proof We set $M:=\left\{F \in \Sigma \mid \varphi(l) F[l]=1 \forall l \in \mathcal{S}_{L}(C)\right\}$. It is easy to see that $\varphi(l)\left\langle F, l l^{\dagger}\right\rangle=1$ is an affine condition on $F$ and therefore $\operatorname{dim}_{\mathbb{R}}(M)$ is in fact the perfection corank of $C$. Obviously $C \subset M$ and therefore Aff $(C) \subset M$ holds. To see the converse let $F \in C$ and let $m_{1}, \ldots, m_{r}$ be a basis for the space of translations of $M$. We may rescale $m_{1}, \ldots, m_{r}$ such that $F+m_{i} \in \mathcal{P}$ for all $i$. Clearly $\left(F+m_{i}\right)[l]=1$ for all $l \in \mathcal{S}_{L}(C)$ and after rescaling the $m_{i}$ again we may also assume that $\left(F+m_{i}\right)[l]>1$ for all $0 \neq l \in L-\mathcal{S}_{L}(C)$. But then $F+m_{i} \in C$ for all $1 \leq i \leq r$ and $C$ contains an affine basis of $M$.

The following corollary is now easy to see.

Corollary 2 The perfection rank is a strictly increasing function on the set of minimal classes with the partial ordering defined above.

Remark 5 Let $C \subset \Sigma_{=1}^{\mathrm{wr}}$ be a minimal class. Then $C$ is bounded (as a subset of $\Sigma$ ).

The last two assertions have prepared us to prove the most important of the structural properties of our cell decomposition. 
Theorem 2 Let $C \subset \Sigma_{=1}^{\mathrm{wr}}$ be a minimal class. Then its closure is the convex hull of the perfect forms it contains.

Proof We will prove this via induction on the perfection corank. If $C$ is the class corresponding to a perfect form there is nothing to show. If $C$ is not the class of a perfect form let $F \in C$ and let $F^{\prime} \in \bar{C}$ be a perfect form. Now we know that $C$ is bounded, hence there is some $\rho>0$ such that $F^{\prime \prime}:=F+\rho\left(F-F^{\prime}\right)$ is in the boundary of $C$. Since $C$ was open in $\operatorname{Aff}(C)$ we have $\mathrm{Cl}_{L}\left(F^{\prime \prime}\right) \varsubsetneqq C$. By induction $F^{\prime \prime}$ is a convex combination of perfect forms in the closure of $C$. Now $F$ is a convex combination of $F^{\prime}$ and $F^{\prime \prime}$ which implies the assertion.

This theorem has some computationally very useful consequences.

Corollary $3 \mathrm{Up}$ to the action of $\mathrm{GL}(L)$ there are only finitely many well-rounded minimal classes.

Corollary 4 If $C \subset \Sigma_{=1}^{\mathrm{wr}}$ is a minimal class there are only finitely many minimal classes contained in $\bar{C}$.

Corollary 5 Let $C \subset \Sigma_{=1}^{\mathrm{wr}}$ be a minimal class. $\bar{C}$ is a bounded polytope whose faces are exactly the minimal classes $C^{\prime} \varsubsetneqq C$.

\section{Homology computations}

The previous section provided us with a finite-dimensional $\mathrm{CW}$-complex together with a cellular GL $(L)$-action. Hence the cellular chain complex arising from the decomposition of $\Sigma_{=1}^{\mathrm{wr}}$ into minimal classes is in fact a chain complex of GL $(L)$-modules. We want to use this information to compute a free $\mathbb{Z}[\mathrm{GL}(L)]$-resolution of $\mathbb{Z}$ which may then be used for homology computations.

\subsection{Perturbations}

First note that the GL( $L)$-modules appearing in the cellular chain complex of $\Sigma_{=1}^{\mathrm{wr}}$ are not free as each cell has a non-trivial stabilizer. Hence the cellular chain complex itself does not constitute a $\mathbb{Z}[\mathrm{GL}(L)]$-free resolution of $\mathbb{Z}$. However the following theorem originally due to Wall [24] allows us to combine the cellular chain complex with free resolutions of $\mathbb{Z}$ over the group rings of stabilizers of minimal classes to obtain the desired resolution. This result is an instance of homological perturbation theory; we refer the reader to $[16,17]$ for a general account. We do not worry here about finding resolutions for the stabilizers as all these groups are finite in which case there are computational methods readily available (see for example [12]).

Theorem 3 [14, Prop. 1, Prop. 4] Let $\left\{A_{p, q} \mid p, q \geq 0\right\}$ be a bigraded family of $\mathbb{Z} G$-free modules und $d_{0}: A_{p, q} \rightarrow A_{p, q-1}$ homomorphisms such that $\left(A_{p, *}, d_{0}\right)$ is an acyclic chain complex for each $p$. We set $C_{p}:=\mathrm{H}_{0}\left(A_{p, *}\right)$ and assume furthermore that there are homomorphisms $\partial: C_{p} \rightarrow C_{p-1}$ such that $\left(C_{*}, \partial\right)$ is a chain complex. Then the following holds: 
1. There are homomorphisms $d_{k}: A_{p, q} \rightarrow A_{p-k, q+k-1}$ for $k \geq 1, p>k$ such that

$$
d=d_{0}+d_{1}+d_{2}+\cdots: R_{n}:=\bigoplus_{p+q=n} A_{p, q} \rightarrow R_{n-1}=\bigoplus_{p+q=n-1} A_{p, q}
$$

is the differential of a chain complex $R_{*}$ of free $\mathbb{Z} G$-modules.

2. The canonical chain map $\phi_{p}: A_{p, *} \rightarrow \mathrm{H}_{0}\left(A_{p, *}\right)$ yields a chain map $\phi_{*}: R_{*} \rightarrow$ $C_{*}$ which induces an isomorphism in homology.

3. Assume that there are $\mathbb{Z}$-homomorphisms $h_{0}: A_{p, q} \rightarrow A_{p, q+1}$ with $d_{0} h_{0} d_{0}(x)=$ $d_{0}(x)$ for all $x \in A_{p, q+1}$ (a so called contracting homotopy). Then we can construct $d_{k}$ by first lifting $\partial$ to $d_{1}: A_{p, 0} \rightarrow A_{p-1,0}$ and setting

$$
d_{k}=-h_{0}\left(\sum_{i=1}^{k} d_{i} d_{k-i}\right)
$$

recursively on the free generators of $A_{p, q}$.

In our situation this reads as follows:

Let $X:=\Sigma_{=1}^{\mathrm{wr}}$ denote the CW-complex of well-rounded forms of minimum 1. For $p \in \mathbb{Z}_{\geq 0}$ let $e_{p}$ be a system of representatives of minimal classes of perfection corank (dimension) $p$. Then

$$
C_{p}(X) \cong \bigoplus_{c \in e_{p}} \mathbb{Z}[\mathrm{GL}(L)] \otimes_{\mathbb{Z}[\operatorname{Stab}(c)]} \mathbb{Z}^{\chi_{c}}
$$

where $\operatorname{Stab}(c):=\operatorname{Stab}_{\mathrm{GL}(L)}(c)$ is the stabilizer of $c$ in $\operatorname{GL}(L)$ acting on $\mathbb{Z}$ via the character $\chi_{c}$ induced by the action of $\operatorname{Stab}(c)$ on the orientation of $c$. Now let $R_{*}^{c}$ be a $\mathbb{Z}[\operatorname{Stab}(c)]$-free resolution of $\mathbb{Z}^{\chi_{c}}$ then

$$
\bigoplus_{c \in e_{p}} \mathbb{Z}[\mathrm{GL}(L)] \otimes_{\mathbb{Z}[\operatorname{Stab}(c)]} R_{*}^{c}
$$

is a $\mathbb{Z}[\mathrm{GL}(L)]$ free resolution of $C_{p}(X)$ and the theorem becomes applicable.

The algorithm to compute a free resolution in this setup is part of the GAP-package "HAP" [13] and needs as input the combinatorial structure of the cell complex and the finite stabilizers.

The above algorithm cannot only be used for homology computations of the full group GL $(L)$ but also for subgroups of finite index in $\operatorname{GL}(L)$ if enough about them is known (e.g. if we can check for membership and know the index in GL $(L)$ ). For example one can do the computations for unit groups of non-maximal orders or special linear groups over imaginary quadratic number fields. Note that if said subgroup is torsion free every stabilizer is trivial and the cellular chain complex is already a free resolution without first applying Wall's perturbation lemma. Furthermore all of the above still holds if we quotient by a subgroup acting trivially on $\mathcal{P}$ (in most cases this will only be $\langle-1\rangle$ ). 


\begin{tabular}{cll}
\cline { 2 - 3 } $\begin{array}{l}\text { Table } 1 \\
\text { of } \operatorname{PSL}_{2}\left(\mathcal{O}_{K}\right) \text { for } K=\mathbb{Q}(\sqrt{-5})\end{array}$ & $n$ & $\mathrm{H}_{n}(\Gamma, \mathbb{Z})$ \\
\cline { 2 - 3 } & 1 & $\mathbb{Z} / 2 \mathbb{Z} \times \mathbb{Z} / 6 \mathbb{Z} \times \mathbb{Z}^{2}$ \\
2 & $\mathbb{Z} / 2 \mathbb{Z} \times \mathbb{Z} / 12 \mathbb{Z} \times \mathbb{Z}$ \\
3 & $(\mathbb{Z} / 2 \mathbb{Z})^{2} \times \mathbb{Z} / 6 \mathbb{Z}$ \\
4 & $(\mathbb{Z} / 2 \mathbb{Z})^{3} \times \mathbb{Z} / 6 \mathbb{Z}$ \\
5 & $(\mathbb{Z} / 2 \mathbb{Z})^{4} \times \mathbb{Z} / 6 \mathbb{Z}$ \\
6 & $(\mathbb{Z} / 2 \mathbb{Z})^{5} \times \mathbb{Z} / 6 \mathbb{Z}$ \\
7 & $(\mathbb{Z} / 2 \mathbb{Z})^{6} \times \mathbb{Z} / 6 \mathbb{Z}$ \\
8 & $(\mathbb{Z} / 2 \mathbb{Z})^{7} \times \mathbb{Z} / 6 \mathbb{Z}$ \\
9 & $(\mathbb{Z} / 2 \mathbb{Z})^{8} \times \mathbb{Z} / 6 \mathbb{Z}$ \\
10 & $(\mathbb{Z} / 2 \mathbb{Z})^{9} \times \mathbb{Z} / 6 \mathbb{Z}$ \\
\hline
\end{tabular}

\section{Computational results}

In this section we want to present some results obtained using the algorithm we described in the previous section.

\subsection{Groups over imaginary quadratic integers}

We start with an example that is meant as a reliability check for our computations since the homology presented here was already computed in [20] and the results presented there match ours in all considered dimensions.

Consider the imaginary quadratic number field $K=\mathbb{Q}(\sqrt{-5})$ and the group $\Gamma:=$ $\operatorname{PSL}_{2}\left(\mathcal{O}_{K}\right)$. We obtain a resolution of $\mathbb{Z}$ over $\mathbb{Z}[\Gamma]$ and compute (Table 1$)$.

If the ring of integers of the imaginary quadratic number field has class number greater than one there are multiple conjugacy classes of maximal orders and the method described here can be used to compute a resolution for each of the unit groups. In many cases this can be used to distinguish between these groups.

Consider the imaginary quadratic number field $\mathbb{Q}(\sqrt{-6})$. Then its ring of integers $\mathcal{O}_{K}=\mathbb{Z}[\sqrt{-6}]$ has class number two and thus there are two isomorphism classes of $\mathcal{O}_{K}$-lattices of dimension two represented by $L_{0}:=\mathcal{O}_{K}^{2}$ and $L_{1}:=\mathcal{O}_{K} \oplus \mathfrak{p}$ where $\mathfrak{p}=\langle 2, \sqrt{-6}\rangle$. Consequently there are two conjugacy classes of maximal orders in $K^{2 \times 2}$ with corresponding unit groups $\operatorname{GL}\left(L_{0}\right)=\mathrm{GL}_{2}\left(\mathcal{O}_{K}\right)$ and $\operatorname{GL}\left(L_{1}\right)$. We compute the integral homology of these two unit groups up to degree 10 and can for example conclude that $\operatorname{GL}\left(L_{0}\right)$ and $\operatorname{GL}\left(L_{1}\right)$ are not isomorphic as abstract groups (Table 2).

For linear groups over imaginary quadratic number fields in dimension higher than 2 only little is known. The only results known to the author appeared in [11] where the authors computed the homology in dimension 3 up to small torsion. Since the complexity of our cell complex grows rapidly with the dimension and apparently with the discriminant of the order (see also Sect. 5.5) we are not capable of computing the homology in these cases up to a high degree, however we can compute the (so far missing) torsion in low degrees. 
Table 2 The integral homology of $\mathrm{GL}_{2}\left(\mathcal{O}_{K}\right)$ and $\operatorname{GL}\left(\mathcal{O}_{K} \oplus \mathfrak{p}\right)$ for $K=\mathbb{Q}(\sqrt{-6})$

\begin{tabular}{lll}
\hline$n$ & $\mathrm{H}_{n}\left(\mathrm{GL}\left(L_{0}\right), \mathbb{Z}\right)$ & $\mathrm{H}_{n}\left(\mathrm{GL}\left(L_{1}\right), \mathbb{Z}\right)$ \\
\hline 1 & $\left(\mathbb{Z} / 2 \mathbb{Z}^{4}\right.$ & $(\mathbb{Z} / 2 \mathbb{Z})^{4}$ \\
2 & $(\mathbb{Z} / 4 \mathbb{Z})^{2} \times \mathbb{Z} / 12 \mathbb{Z} \times \mathbb{Z}$ & $(\mathbb{Z} / 2 \mathbb{Z})^{2} \times \mathbb{Z} / 12 \mathbb{Z} \times \mathbb{Z}$ \\
3 & $(\mathbb{Z} / 2 \mathbb{Z})^{9} \times \mathbb{Z} / 24 \mathbb{Z}$ & $(\mathbb{Z} / 2 \mathbb{Z})^{8} \times \mathbb{Z} / 24 \mathbb{Z}$ \\
4 & $(\mathbb{Z} / 2 \mathbb{Z})^{7}$ & $(\mathbb{Z} / 2 \mathbb{Z})^{6} \times \mathbb{Z} / 4 \mathbb{Z}$ \\
5 & $(\mathbb{Z} / 2 \mathbb{Z})^{13}$ & $(\mathbb{Z} / 2 \mathbb{Z})^{13}$ \\
6 & $(\mathbb{Z} / 2 \mathbb{Z})^{8} \times(\mathbb{Z} / 4 \mathbb{Z})^{2} \times \mathbb{Z} / 12 \mathbb{Z}$ & $(\mathbb{Z} / 2 \mathbb{Z})^{10} \times \mathbb{Z} / 12 \mathbb{Z}$ \\
7 & $(\mathbb{Z} / 2 \mathbb{Z})^{17} \times \mathbb{Z} / 24 \mathbb{Z}$ & $(\mathbb{Z} / 2 \mathbb{Z})^{16} \times \mathbb{Z} / 24 \mathbb{Z}$ \\
8 & $(\mathbb{Z} / 2 \mathbb{Z})^{15}$ & $(\mathbb{Z} / 2 \mathbb{Z})^{14} \times \mathbb{Z} / 4 \mathbb{Z}$ \\
9 & $(\mathbb{Z} / 2 \mathbb{Z})^{21}$ & $(\mathbb{Z} / 2 \mathbb{Z})^{21}$ \\
10 & $(\mathbb{Z} / 2 \mathbb{Z})^{16} \times(\mathbb{Z} / 4 \mathbb{Z})^{2} \times \mathbb{Z} / 12 \mathbb{Z}$ & $(\mathbb{Z} / 2 \mathbb{Z})^{18} \times \mathbb{Z} / 12 \mathbb{Z}$ \\
\hline
\end{tabular}

We consider the imaginary quadratic number field $K=\mathbb{Q}(\sqrt{-7})$ and the group $\Gamma:=\mathrm{GL}_{3}\left(\mathcal{O}_{K}\right)$. Using our method we can compute the integral homology up to dimension 3 including torsion.

$$
\mathrm{H}_{n}(\Gamma, \mathbb{Z})= \begin{cases}\mathbb{Z} / 2 \mathbb{Z} & n=1 \\ (\mathbb{Z} / 2 \mathbb{Z})^{3} & n=2 \\ (\mathbb{Z} / 2 \mathbb{Z})^{4} \times(\mathbb{Z} / 4 \mathbb{Z}) \times(\mathbb{Z} / 12 \mathbb{Z})^{2} \times \mathbb{Z}^{2} & n=3\end{cases}
$$

For $K=\mathbb{Q}(\sqrt{-1})$ and $\Gamma=\mathrm{GL}_{3}\left(\mathcal{O}_{K}\right)$ we compute the homology up to dimension 2.

$$
\mathrm{H}_{n}(\Gamma, \mathbb{Z})= \begin{cases}\mathbb{Z} / 4 \mathbb{Z} & n=1 \\ (\mathbb{Z} / 2 \mathbb{Z})^{2} & n=2\end{cases}
$$

Note that these results (in particular the dimensions of the free parts) are compatible with those of [11].

\subsection{Groups over real quadratic fields}

Consider the groups $\mathrm{GL}_{2}\left(\mathcal{O}_{K}\right)$ where $K$ is one of the real quadratic fields $\mathbb{Q}(\sqrt{2})$ and $\mathbb{Q}(\sqrt{3})$. The groups of this type are closely related to the so-called Hilbert modular groups. We compute the integral homology up to dimension 8 and 7 , respectively, and obtain (Table 3).

Again we can also consider the case of a number field whose ring of integers is not a principal ideal domain. For $K=\mathbb{Q}(\sqrt{10})$ there are two isomorphism classes of $\mathcal{O}_{K}$-modules of dimension 2 represented by $L_{0}=\mathcal{O}_{K}^{2}$ and $L_{1}=\mathcal{O}_{K} \oplus \mathfrak{p}$ where $\mathfrak{p}=\langle 2, \sqrt{10}\rangle$. As was the case for $\mathbb{Q}(\sqrt{-6})$, the integral homology is sufficient to distinguish between the two groups $\operatorname{GL}\left(L_{0}\right)$ and $\operatorname{GL}\left(L_{1}\right)$ (Table 4$)$. 
Table 3 The integral homology of $\mathrm{GL}_{2}\left(\mathcal{O}_{K}\right)$ for $K=\mathbb{Q}(\sqrt{2}), \mathbb{Q}(\sqrt{3})$

\begin{tabular}{lll}
\hline$n$ & $\mathrm{H}_{n}\left(\mathrm{GL}_{2}(\mathbb{Z}[\sqrt{2}]), \mathbb{Z}\right)$ & $\mathrm{H}_{n}\left(\mathrm{GL}_{2}(\mathbb{Z}[\sqrt{3}]), \mathbb{Z}\right)$ \\
\hline 1 & $(\mathbb{Z} / 2 \mathbb{Z})^{2} \times \mathbb{Z}$ & $(\mathbb{Z} / 2 \mathbb{Z})^{2} \times \mathbb{Z}$ \\
2 & $(\mathbb{Z} / 2 \mathbb{Z})^{6}$ & $(\mathbb{Z} / 2 \mathbb{Z})^{5} \times \mathbb{Z}$ \\
3 & $(\mathbb{Z} / 2 \mathbb{Z})^{7} \times \mathbb{Z} / 48 \mathbb{Z}$ & $(\mathbb{Z} / 2 \mathbb{Z})^{6} \times(\mathbb{Z} / 24 \mathbb{Z})^{2} \times \mathbb{Z}$ \\
4 & $(\mathbb{Z} / 2 \mathbb{Z})^{11} \times \mathbb{Z} / 24 \mathbb{Z}$ & $(\mathbb{Z} / 2 \mathbb{Z})^{10} \times \mathbb{Z} / 12 \mathbb{Z} \times \mathbb{Z} / 24 \mathbb{Z}$ \\
5 & $(\mathbb{Z} / 2 \mathbb{Z})^{13} \times \mathbb{Z} / 4 \mathbb{Z}$ & $(\mathbb{Z} / 2 \mathbb{Z})^{13} \times \mathbb{Z} / 4 \mathbb{Z}$ \\
6 & $(\mathbb{Z} / 2 \mathbb{Z})^{18}$ & $(\mathbb{Z} / 2 \mathbb{Z})^{18}$ \\
7 & $(\mathbb{Z} / 2 \mathbb{Z})^{19} \times \mathbb{Z} / 48 \mathbb{Z}$ & $(\mathbb{Z} / 2 \mathbb{Z})^{18} \times(\mathbb{Z} / 24 \mathbb{Z})^{2}$ \\
8 & $(\mathbb{Z} / 2 \mathbb{Z})^{23} \times \mathbb{Z} / 24 \mathbb{Z}$ & \\
\hline
\end{tabular}

Table 4 The integral homology of $\mathrm{GL}_{2}\left(\mathcal{O}_{K}\right)$ and $\mathrm{GL}\left(\mathcal{O}_{K} \oplus \mathfrak{p}\right)$ for $K=\mathbb{Q}(\sqrt{10})$

\begin{tabular}{lll}
\hline$n$ & $\mathrm{H}_{n}\left(\mathrm{GL}\left(L_{0}\right), \mathbb{Z}\right)$ & $\mathrm{H}_{n}\left(\mathrm{GL}\left(L_{1}\right), \mathbb{Z}\right)$ \\
\hline 1 & $(\mathbb{Z} / 2 \mathbb{Z})^{2} \times \mathbb{Z}$ & $(\mathbb{Z} / 2 \mathbb{Z})^{2} \times \mathbb{Z}$ \\
2 & $(\mathbb{Z} / 2 \mathbb{Z})^{8} \times \mathbb{Z}$ & $(\mathbb{Z} / 2 \mathbb{Z})^{7} \times \mathbb{Z}$ \\
3 & $(\mathbb{Z} / 2 \mathbb{Z})^{12} \times \mathbb{Z} / 12 \mathbb{Z} \times \mathbb{Z} / 24 \mathbb{Z} \times \mathbb{Z}$ & $(\mathbb{Z} / 2 \mathbb{Z})^{11} \times \mathbb{Z} / 6 \mathbb{Z} \times \mathbb{Z} / 24 \mathbb{Z} \times \mathbb{Z}$ \\
4 & $(\mathbb{Z} / 2 \mathbb{Z})^{19} \times(\mathbb{Z} / 12 \mathbb{Z})^{2}$ & $(\mathbb{Z} / 2 \mathbb{Z})^{18} \times \mathbb{Z} / 6 \mathbb{Z} \times \mathbb{Z} / 12 \mathbb{Z}$ \\
5 & $(\mathbb{Z} / 2 \mathbb{Z})^{24} \times(\mathbb{Z} / 4 \mathbb{Z})^{2}$ & $(\mathbb{Z} / 2 \mathbb{Z})^{24} \times \mathbb{Z} / 4 \mathbb{Z}$ \\
6 & $(\mathbb{Z} / 2 \mathbb{Z})^{32} \times \mathbb{Z} / 4 \mathbb{Z}$ & $(\mathbb{Z} / 2 \mathbb{Z})^{32}$ \\
7 & $(\mathbb{Z} / 2 \mathbb{Z})^{36} \times \mathbb{Z} / 12 \mathbb{Z} \times \mathbb{Z} / 24 \mathbb{Z}$ & $(\mathbb{Z} / 2 \mathbb{Z})^{35} \times \mathbb{Z} / 6 \mathbb{Z} \times \mathbb{Z} / 24 \mathbb{Z}$ \\
8 & $(\mathbb{Z} / 2 \mathbb{Z})^{43} \times(\mathbb{Z} / 12 \mathbb{Z})^{2}$ & $(\mathbb{Z} / 2 \mathbb{Z})^{42} \times \mathbb{Z} / 6 \mathbb{Z} \times \mathbb{Z} / 12 \mathbb{Z}$ \\
\hline
\end{tabular}

\subsection{A quaternion order}

While we mainly used the described algorithms to work out the homology of Bianchi groups, in principle they work in a very general setup, e.g. for unit groups of maximal orders in quaternion algebras. Let $A=\left(\frac{2,3}{\mathbb{Q}}\right)$ be the rational quaternion algebra ramified at 2 and 3 and $\mathfrak{M}_{2,3}$ be a maximal order in $A$. Then $\mathfrak{M}_{2,3}^{\times}$has periodic integral homology (since $\langle-1\rangle$ is the only torsion) and we obtain for $n \geq 1$ :

$$
\mathrm{H}_{n}\left(\mathfrak{M}_{2,3}^{\times}, \mathbb{Z}\right)=\left\{\begin{array}{lll}
\mathbb{Z} / 24 \mathbb{Z} & n \equiv 1 & (\bmod 2) \\
\mathbb{Z} / 2 \mathbb{Z} & n \equiv 0 & (\bmod 2)
\end{array}\right.
$$

In [6] one can find an algorithm to compute a presentation of the unit groups we consider. One of the explicit example given is

$$
\mathfrak{M}_{2,3}^{\times} /\{ \pm 1\} \cong\left\langle a, b, t \mid a^{3}, b^{2}, a t b t\right\rangle
$$


This information can also be used to study the integral homology (cf. [3]) and an implementation is available in the software package MAGNUS (cf. [2]). We compared the results obtained from the presentation and from our implementation and are happy to report that both yield the same integral homology, namely:

$$
\mathrm{H}_{n}\left(\mathfrak{M}_{2,3}^{\times} /\{ \pm 1\}, \mathbb{Z}\right)=\left\{\begin{array}{lll}
\mathbb{Z} / 12 \mathbb{Z} & n=1 \\
\mathbb{Z} / 6 \mathbb{Z} & n \equiv 1 \quad(\bmod 2), n>1 \\
\{0\} & n \equiv 0 & (\bmod 2)
\end{array}\right.
$$

The presentations for the other groups considered in this section appear to be to complicated for MAGNUS to compute the integral homology from them.

\subsection{Additional examples}

For imaginary quadratic number fields $K=\mathbb{Q}(\sqrt{-d})$ with $1 \leq d \leq 26$ we computed resolutions for the groups $\operatorname{GL}(L)$ and $\operatorname{SL}(L)$ where $L$ runs through a system of representatives of isomorphism classes of lattices in $K^{2}$ (whence End $\mathcal{O}_{K}(L)$ runs through a system of representatives of conjugacy classes of maximal orders). These complexes are freely available in the GAP-package HAP $[13,15]$ and on the author's homepage.

For imaginary quadratic number fields $K=\mathbb{Q}(\sqrt{-d})$ with $d \in\{1,2,3,7,11,15\}$ we computed contractible complexes for the groups $\mathrm{GL}_{3}\left(\mathcal{O}_{K}\right)$. These complexes are available from the author's homepage in HAP-readable format.

For real quadratic number fields $K=\mathbb{Q}(\sqrt{d})$ with $2 \leq d \leq 15$ squarefree we computed contractible complexes for the groups $\mathrm{GL}(L)$ where $L$ runs through a system

Table 5 The well-rounded complexes of $\mathrm{GL}_{2}$ and $\mathrm{GL}_{3}$ over imaginary quadratic integers

\begin{tabular}{llcll}
\hline$D$ & Nbr. of cells & Max. bound. & Max. stab. & Runtime \\
\hline$n=2$ & & & & \\
3 & {$[1,1,1]$} & 6 & Order 72 solvable & $<1 \mathrm{~s}$ \\
4 & {$[1,1,1]$} & 4 & Order 96 solvable & $<1 \mathrm{~s}$ \\
7 & {$[1,2,1]$} & 6 & Order 12 solvable & $<1 \mathrm{~s}$ \\
8 & {$[1,2,1]$} & 4 & Order 48 solvable & $<1 \mathrm{~s}$ \\
11 & {$[1,2,1]$} & 6 & Order 24 solvable & $<1 \mathrm{~s}$ \\
15 & {$[2,4,4]$} & 6 & Order 12 solvable & $<1 \mathrm{~s}$ \\
$n=3$ & & & & \\
3 & {$[2,2,3,4,3,2,1]$} & 54 & Order 1296 solvable & $\sim 4.5 \mathrm{~min}$ \\
4 & {$[1,1,3,5,4,3,2]$} & 76 & Order 348 solvable & $\sim 1 \mathrm{~min}$ \\
7 & {$[2,2,8,11,9,6,3]$} & 70 & $C_{2} \times$ PSL 3 (2) & $\sim 1 \mathrm{~min}$ \\
8 & {$[2,7,28,37,26,16,5]$} & 220 & Order 96 solvable & $\sim 6 \mathrm{~min}$ \\
11 & {$[12,51,125,150,91,34,8]$} & 478 & Order 48 solvable & $\sim 59 \mathrm{~min}$ \\
15 & {$[11,86,299,454,338,137,28]$} & 474 & Order 48 solvable & $\sim 16 \mathrm{~h}$ \\
\hline
\end{tabular}


of representatives of the $\mathcal{O}_{K}$-isomorphism classes of modules of dimension 2 . These complexes are also available from the author's homepage in HAP-readable format.

\subsection{Sizes of the cell complexes}

As previously noted the complexity of our cell complex appears to increase with growing discriminant. To illustrate this and to give a picture of the scaling of the algorithms we give some information in the following table. We consider the groups of type $\mathrm{GL}_{2}\left(\mathcal{O}_{K}\right)$ and $\mathrm{GL}_{3}\left(\mathcal{O}_{K}\right)$ with $K=\mathbb{Q}(\sqrt{-D})$ where $-D$ is the discriminant of $\mathcal{O}_{K}$. These groups have virtual cohomological dimension 2 and 6, respectively, and we present the number of pairwise inequivalent cells in all relevant dimensions, the maximal number of cells appearing in the boundary of a cell (which is always realized at a cell of maximal dimension), some information on the largest appearing stabilizer, and the time it took to construct the full complex (i.e. without applying Wall's lemma). The computations were performed on an Intel core i7 processor running at $2.93 \mathrm{GHz}$ (Table 5).

Acknowledgements The author is supported by the DFG Research training Group Experimental and constructive algebra (GRK 1632). The author is very thankful to the mathematics department at NUI Galway, and Alexander Rahm in particular, for their hospitality during his stay in Galway in fall 2013. The results presented here are part of the author's Master's thesis which was supervised by Gabriele Nebe.

\section{References}

1. Ash, A.: Small-dimensional classifying spaces for arithmetic subgroups of general linear groups. Duke Math. J. 51(2), 459-468 (1984)

2. Baumslag, G., Miller, C.F., III: Experimenting and computing with infinite groups. In: Groups and Computation, II (New Brunswick, NJ, 1995), DIMACS Ser. Discrete Math. Theoret. Comput. Sci., vol. 28, American Mathematical Society, Providence, RI, pp. 19-30 (1997)

3. Baumslag, G., Dyer, E., Miller, C.F.: On the integral homology of finitely presented groups. Bull Am. Math. Soc. (NS) 4(3), 321-324 (1981). doi:10.1090/S0273-0979-1981-14898-9

4. Berkove, E.: The mod-2 cohomology of the Bianchi groups. Trans. Am. Math. Soc. 352(10), 45854602 (2000)

5. Bosma, W., Cannon, J., Playoust, C.: The Magma algebra system. I. The user language. J. Symb. Comput. 24(3-4), 235-265 (1997). computational algebra and number theory (London, 1993)

6. Braun, O., Coulangeon, R., Nebe, G., Schönnenbeck, S.: Computing in arithmetic groups with Voronoï's algorithm. J. Algebra 435, 263-285 (2015)

7. Coulangeon, R.: Invariants d'Hermite, théorie de Voronoï et designs sphériques, habilitation thesis, University of Bordeaux (2004). http://www.math.u-bordeaux1.fr/ rcoulang/synthese.pdf

8. Coulangeon, R., Nebe, G.: Maximal finite subgroups and minimal classes. Enseign Math. 60(3-4), 231-246 (2014)

9. Cremona, J.E.: Hyperbolic tessellations, modular symbols, and elliptic curves over complex quadratic fields. Compos. Math. 51(3), 275-324 (1984)

10. Dutour Sikirić, M., Ellis, G., Schürmann, A.: On the integral homology of $\mathrm{PSL}_{4}(\mathbb{Z})$ and other arithmetic groups. J. Number Theory 131(12), 2368-2375 (2011)

11. Dutour Sikirić, M., Gangl, H., Gunnells, P.E., Hanke, J., Schürmann, A., Yasaki, D.: On the cohomology of linear groups over imaginary quadratic fields. J. Pure Appl. Algebra 220(7), 2564-2589 (2016)

12. Ellis, G.: Computing group resolutions. J. Symb. Comput. 38(3), 1077-1118 (2004)

13. Ellis, G.: HAP - homological algebra programming, a package for the GAP computer algebra system (2013). http://hamilton.nuigalway.ie/Hap/www/ 
14. Ellis, G., Harris, J., Sköldberg, E.: Polytopal resolutions for finite groups. J. Reine Angew. Math. 598, 131-137 (2006)

15. GAP: GAP — groups, algorithms, and programming, Version 4.8.4. The GAP Group (2016). http:// www.gap-system.org

16. Huebschmann, J: On the construction of $A_{\infty}$-structures. Georgian Math. J. 17(1):161-202 (2010). arxiv:0809.4791 [math.AG]

17. Huebschmann, J.: Origins and breadth of the theory of higher homotopies. In: Higher Structures in Geometry and Physics, Progr. Math., vol. 287, Birkhäuser/Springer, New York, pp 25-38 (2011). arxiv:0710.2645 [math.AT]

18. Koecher, M.: Beiträge zu einer Reduktionstheorie in Positivitätsbereichen. I. Math. Ann. 141, 384-432 (1960)

19. Opgenorth, J.: Dual cones and the Voronoi algorithm. Exp. Math. 10(4), 599-608 (2001)

20. Rahm, A.D., Fuchs, M.: The integral homology of $\mathrm{PSL}_{2}$ of imaginary quadratic integers with nontrivial class group. J. Pure Appl. Algebra 215(6), 1443-1472 (2011)

21. Reiner, I.: Maximal Orders, vol. 38. Academic Press, London (1975)

22. Schwermer, J., Vogtmann, K.: The integral homology of $\mathrm{SL}_{2}$ and $\mathrm{PSL}_{2}$ of Euclidean imaginary quadratic integers. Comment. Math. Helv. 58(4), 573-598 (1983)

23. Soulé, C.: The cohomology of $\mathrm{SL}_{3}(Z)$. Topology 17(1), 1-22 (1978)

24. Wall, C.T.C.: Resolutions for extensions of groups. Proc. Camb. Philos. Soc. 57, 251-255 (1961) 